SUBJECT AREAS:

CELL DEATH AND IMMUNE RESPONSE

CANCER

Received

31 March 2014

Accepted

31 July 2014

Published

20 August 2014

Correspondence and requests for materials should be addressed to

C.M. (jq1001@ujs. edu.cn) or D.C. (cdeyu@hotmail.com)

* These authors contributed equally to this work.

\title{
The Regulatory Effect of UL-16 Binding Protein-3 Expression on the Cytotoxicity of NK Cells in Cancer Patients
}

\author{
Xiao Mou ${ }^{1,2 *}$, Yuepeng Zhou ${ }^{1,2 *}$, Peng Jiang ${ }^{3 *}$, Tong Zhou ${ }^{4}$, Qian Jiang ${ }^{1}$, Chengcheng Xu ${ }^{1,2}$, Hongli Liv ${ }^{1,2}$, \\ Tingting Zheng ${ }^{1,2}$, Guoyue Yuan', Yanyun Zhang ${ }^{5}$, Deyu Chen ${ }^{1,2}$ \& Chaoming Mao ${ }^{1,2}$
}

\begin{abstract}
'Department of Nuclear Medicine, The Hospital Affiliated to Jiangsu University, Zhenjiang, China, ${ }^{2}$ Institute of Oncology, The Hospital Affiliated to Jiangsu University, Zhenjiang, China, ${ }^{3}$ Department of anesthesiology, The Hospital Affiliated to Jiangsu University, Zhenjiang, China, ${ }^{4}$ Department of Pediatrics, Ruijin Hospital, Shanghai Jiao Tong University School of Medicine, Shanghai, China, ${ }^{5}$ Key Laboratory of Stem Cell Biology, Institute of Health Sciences, Shanghai Institutes for Biological Sciences, Chinese Academy of Sciences, Shanghai, China.
\end{abstract}

The activating immunoreceptor NKG2D (natural killer group 2, member D) and its ligands play important roles in the innate and adaptive immune responses. UL16-binding protein 3 (ULBP3), an NKG2D ligand, is overexpressed on certain epithelial tumor cells. In this study, we investigated the effect of ULBP3 expression on the cytotoxic activity of natural killer (NK) cells. ULBP3 were measured by flow cytometry analysis, immunohistochemistry, and time-resolved fluoroimmunoassay. The cytotoxicity of NK cells was determined with the lactate dehydrogenase release assay. We found that ULBP3 was overexpressed on tumor cell lines and tumor tissues. Serum from cancer patients, but not from healthy donors, contained elevated levels of soluble ULBP3 (sULBP3). Importantly, high expression of ULBP3 on the cell surface of tumor cells augmented NKG2D-mediated NK cell cytotoxicity. However, low levels of sULBP3 $(<15 \mathrm{ng} / \mathrm{ml}$ ) weakened the cytotoxicity of NK cells by decreasing NKG2D expression on NK cells. Further analysis showed that serum samples from most cancer patients $(>70 \%)$ contained the low level of sULBP3. Our results demonstrate that tumor cells express surface and soluble ULBP3, which regulate NK cell activity. Thus, ULBP3 is a potential therapeutic target for improving the immune response against cancer.

$\mathrm{N}$ atural killer (NK) cells, components of the innate immune system, contribute to the elimination of virusinfected cells as well as to antitumor immune responses ${ }^{1}$. NK cell reactivity is guided by the principles of "missing-self" and "induced-self," in which NK cells are activated by the downregulation or absence of major histocompatibility complex (MHC) I expression ("missing-self") and/or by the stress-induced expression of ligands that bind activating NK receptors ("induced-self"). The balance of various activating and inhibitory signals determines whether NK cell responses are initiated ${ }^{2-5}$.

Among the activating NK receptors, NKG2D (natural killer group 2, member D) is particularly relevant for tumor cell recognition and killing. NKG2D is a C-type lectin-like activating receptor expressed on the cell surface of almost all NK cells, some cytotoxic CD8 ${ }^{+} \alpha \beta \mathrm{T}$ cells, NK T cells, and $\gamma \delta$ T cells, and a small subset of CD $4^{+} \alpha \beta \mathrm{T}$ cell $^{6-8}$. NKG2D mediates NK cell activation by overcoming inhibitory signals from self recognition ${ }^{9,10}$. Malignant transformation induces the expression of NKG2D ligands (NKG2DL), as documented in a variety of human and mouse tumors. The activating immunoreceptor NKG2D endows cytotoxic lymphocytes with the capacity to recognize and eliminate malignant cells, and it plays a critical role in immune surveillance ${ }^{11}$. For example, NKG2DL-expressing tumor cells grafts were efficiently rejected, whereas parental NKG2D-ligand negative tumor cells formed tumors ${ }^{12,13}$. A distinctive feature of the NKG2D recognition system is that NKG2D can interact with a number of distinct ligands with affinities ranging from 4 to $400 \mathrm{nM}^{14-16}$. The ligands recognized by NKG2D, which belong to distinct and relatively distantly related families, include major histocompatibility complex class-I related chain (MIC) A, MICB, and UL16-binding proteins (ULBPs) in humans ${ }^{10,17}$. NKG2DLs are generally not expressed on benign cells, but are induced by cellular stress, genotoxic stress, and infection ${ }^{18,19}$. The human ULBP proteins are widely expressed by various tumor types, including leukemia, and primary solid tumors ${ }^{20-22}$.

In addition to expressing NKG2DLs on their surface, tumors spontaneously release soluble ligands ${ }^{23}$. Soluble MICA secreted by tumor cells downregulated surface NKG2D expression on T cells to induce the functional impairment of anti-tumor immune effector cells, suggesting that shedding may reduce the expression of 
NKG2DLs on the tumor cell surface and contribute to tumor escape from immunosurveillance. Soluble MICA induced the internalization and lysosomal degradation of the NKG2D receptor in $\mathrm{CD}^{+} \mathrm{T}$ and NK cells ${ }^{24-26}$, further reducing the efficiency of NKG2D recognition. Elevated serum levels of soluble MICA have been detected in patients with various types of cancer and may represent a diagnostic marker in patients with suspected malignancies ${ }^{27,28}$.

Unlike other NKG2DLs, ULBP3 has a moderate affinity for NKG2D. However, the regulatory function of ULBP3 in NK cells and its significance in cancer patients are largely unknown. In the present study, ULBP3 expression in several tumor cell lines and tumor tissue cells from common cancer types was analyzed. The effects of surface and soluble forms of ULBP3 on the interaction between tumor cells and NK cells were examined. Our results showed that ULBP3 regulated the activity of NK cells against tumors. Thus, ULBP3 provides a target for tumor immunotherapy.

\section{Results}

Elevated expression of ULBP3 in tumor cell lines and tumor tissues. To evaluate the distribution of the NKG2DL ULBP3 in tumor cells from common cancers, the surface expression of ULBP3 in SW620, K562, 7721, A549, and ECA109 cell lines was analyzed by flow cytometry (FCM) analysis. The colorectal cancer cell line $\mathrm{CD} 133^{-} \mathrm{SW} 620$ expressed high levels $(>50 \%)$ of ULBP3 $(59.0 \pm 2.6 \%, \mathrm{n}=3)$, and $\mathrm{CD} 1333^{+} \mathrm{SW} 620$ cells expressed moderate levels $(20 \%-50 \%)$ of ULBP3 $(22.0 \pm 1.4 \%, \mathrm{n}=3)$. The liver cancer cell line 7721 also expressed a moderate level of ULBP3 protein $(30.0 \pm 3.7 \%, \mathrm{n}=3)$. However, surface ULBP3 protein was undetectable on the lung cancer cell line A549 and esophageal carcinoma cell line ECA109. The leukemic cell line K562, which does not express surface ULBP3, was used as a negative control (Figure 1A). We then examined the expression of ULBP3 in different tumor tissues. In cancer patients with colorectal cancer ( $\mathrm{n}$ $=5)$, liver cancer $(\mathrm{n}=3)$, lung cancer $(\mathrm{n}=3)$, and gastric cancer $(\mathrm{n}$ $=7$ ), FCS indicated that ULBP3 expression was much higher in the tumor tissue than in the adjacent non-tumor tissue (ANTT). Representative dot graphs are shown in Figure 1B. Immunohistochemistry results also showed that ULBP3 expression was upregulated in colorectal $(n=5)$, liver $(n=3)$, lung $(n=5)$, and gastric $(n=5)$ cancer tissue (Figure 1C). The results of RT-PCR analysis demonstrated that the ULBP3 gene was expressed in ULBP3-expressing tumor cell lines and in fresh tumor tissues from cancer patients, but not in ANTT (Figure 1D).

Spontaneous release of soluble ULBP3 (sULBP3) from tumor cell lines and elevated serum SULBP3 in cancer patients. To determine whether tumor cells release sULBP3, we used a time-resolved fluoroimmunoassay (TRFIA) with a $\mathrm{Eu}^{3+}$-labeled anti-ULBP3 monoclonal antibody. The detection limit of the TRFIA method for ULBP3-Fc was approximately $0.05 \mathrm{ng} / \mathrm{ml}$ (Figure 2A). After $48 \mathrm{~h}$ of culture, sULBP3 levels in the supernatants of SW620, K562, 7721, A549, and ECA109 cells were measured. No sULBP3 was detectable in the culture supernatants of K562, A549, and ECA109 cells or in the culture supernatants of resting or activated NK cells. However, the levels of sULBP3 were detected in the supernatants of the tumor cell lines CD133 ${ }^{+}$SW620 (3.6 \pm $0.08 \mathrm{ng} / \mathrm{ml}), \mathrm{CD}_{133}{ }^{-} \mathrm{SW} 620(6.0 \pm 0.1 \mathrm{ng} / \mathrm{ml})$, and $7721(4.3 \pm$ $0.1 \mathrm{ng} / \mathrm{ml}$ ) (Figure 2B). To determine whether ULBP3 was released by human tumors in vivo, we analyzed serum sULBP3 levels in 116 patients with various malignancies and 48 healthy volunteers. Compared with healthy volunteers $(1.5 \pm 0.1 \mathrm{ng} / \mathrm{ml})$, the serum concentration of sULBP3 was significantly higher in cancer patients (colorectal cancer: $\mathrm{n}=45,14.4 \pm 2.5 \mathrm{ng} / \mathrm{ml}, P<0.001$; gastric cancer: $\mathrm{n}=38,8.4 \pm 1.1 \mathrm{ng} / \mathrm{ml}, P<0.001$; lung cancer: $\mathrm{n}=$ $33,9.6 \pm 1.4 \mathrm{ng} / \mathrm{ml}, P<0.001$ ) (Figure $2 \mathrm{C}$ ). Further analysis showed that $73.3 \%$ of colorectal cancer patients, $83.3 \%$ of gastric cancer patients, and $82.3 \%$ of lung cancer patients were distributed in the low level of serum ULBP3 $(<15 \mathrm{ng} / \mathrm{ml})$, as shown in Table 1 .

Differences in the functional effects of surface ULBP3 and sULBP3 on NK cell activity. Next, we assessed the effect of ULBP3 cell surface expression on the lytic capacity of effector NK cells. CD133 ${ }^{+}$SW620 (moderate expression of surface ULBP3), CD133- ${ }^{-}$W620 (high expression of surface ULBP3), and CD133-SW620 ${ }^{-}$ULBP3-siRnA $(\mathrm{no} /$ low expression of surface ULBP3; Figure 3A) tumor cells were used as target cells, and $\mathrm{NK}$ cells were freshly isolated from healthy volunteers for use as effector cells in cytotoxicity assays. With CD133-SW620 ${ }^{\text {ULBP3-siRNA }}$ cells, the efficiency of NK-cell mediated killing was low when the effector cell to target cell $(\mathrm{E}: \mathrm{T})$ ratio was low $(5: 1$ or $1: 1)$ or high $(10: 1)$. However, a high level of cytotoxicity was observed with $\mathrm{CD}_{133^{-}}$SW620 cells, and a moderate level of cytotoxicity was found with $\mathrm{CD}_{133}{ }^{+} \mathrm{SW} 620$ cells. These results suggested that the amount of ULBP3 expressed on the cell surface of tumor cells affected the cytotoxicity of NK cells (Figure 3B). In these experiments, blocking NKG2D on the surface of NK cells with a specific neutralizing monoclonal antibody strongly reduced the cytolytic activity of NK cells against the target cells at an E:T ratio of $10: 1$, indicating that the NKG2D/NKG2DL pathway was involved in modulating the cytotoxic activity of NK cells (Figure 3C).

To determine the effect of sULBP3 on the lytic capacity of NK cells, different concentrations of soluble recombinant ULBP3-Fc were added to the culture medium of tumor cells and NK cells, and cytotoxicity was measured. In the absence of surface ULBP3 expression on K562 and A549 cells, the addition of a low dose of sULBP3 $(<15 \mathrm{ng} / \mathrm{ml})$ reduced NK cell-mediated cytotoxicity against K562 cells at an E:T ratio of $10: 1$. However, no significant increase in NK cell cytotoxicity was observed when high concentrations of sULBP3 $(>15 \mathrm{ng} / \mathrm{ml})$ were added to co-culture of NK and target cells (Figure 4A and 4B). With 7721 and CD133 ${ }^{-}$SW620 cells expressing moderate to high levels of surface ULBP3, similar results were observed (Figure 4C and 4D). Then, we studied the effect of soluble ULBP3 on the lytic capacity of NK cells on different E: T ratios, we found that, firstly, in the non-expression of surface ULBP3 on K562 and A549 target cells, the presence of low dose of sULBP3 (1 ng/ml) caused a statistically reduction of NK cell cytotoxicity against target cells at $\mathrm{E}: \mathrm{T}$ ratio of $5: 1$ and $10: 1$. However, no significant increase of NK cell cytotoxicity was observed when high concentration of sULBP3 $(30 \mathrm{ng} / \mathrm{ml})$ was added in co-culture of NK and target cells (Figure 4E and 4F). Secondly, in the moderate/high expression of surface ULBP3 on 7721 and CD133-SW620 target cells, the similar results were observed (Figure $4 \mathrm{G}$ and $4 \mathrm{H}$ ).

To confirm that sULBP3 regulated NK cell-mediated cytotoxicity, we incubated sULBP3-containing supernatants for $30 \mathrm{~min}$ in the absence or presence of an NKG2D-Fc fusion protein $(5 \mathrm{ng} / \mathrm{ml})$ before adding the supernatants to co-culture of NK cells and tumor cells. Neutralization of sULBP3 by NKG2D-Fc diminished the inhibitory effect of the sULBP3-containg supernatants on NK cell cytotoxicity against K562 (Figure 5A), 7721 (Figure 5B), and CD133 ${ }^{-}$SW620 (Figure 5C) cells, and no significant effects were detected in the NKp44-Fc control group.

To determine the mechanism by which sULBP3 affects the activity of NK cells, NK cells were pre-incubated with different concentrations of ULBP3-Fc for $6 \mathrm{~h}$, and NKG2D expression was measured. When ULBP3-Fc was added to the culture medium, NKG2D expression on NK cells decreased, particularly with $1 \mathrm{ng} / \mathrm{ml}$ ULBP3-Fc. A representative graph depicting NKG2D expression is shown in Figure 5D, and combined data are shown in Figure 5E.

An anti-ULBP3 monoclonal antibody enhances the activity of NK cells from cancer patients via antibody-dependent cell-mediated cytotoxicity (ADCC). To investigate the relationship between the level of ULBP3 expression on tumor cells and the number of infiltrating NK cells in cancer tissues, we measured ULBP3 levels 

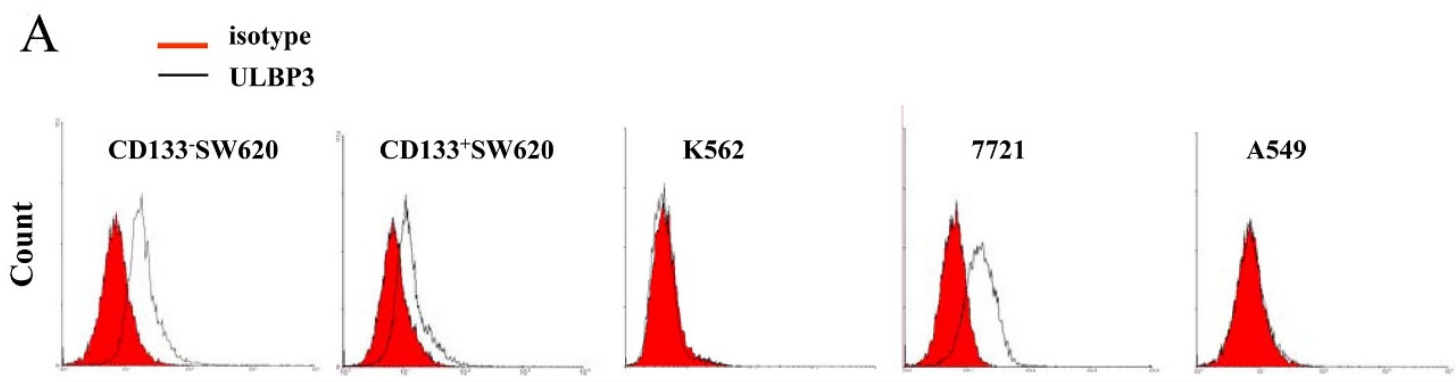

ECA109

\section{ULBP3}

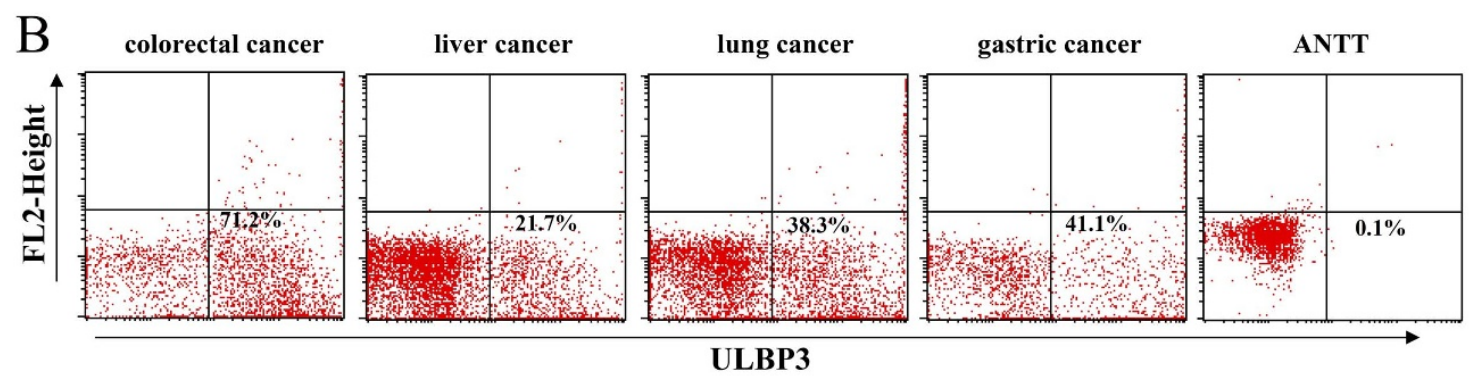

$\mathrm{C}$
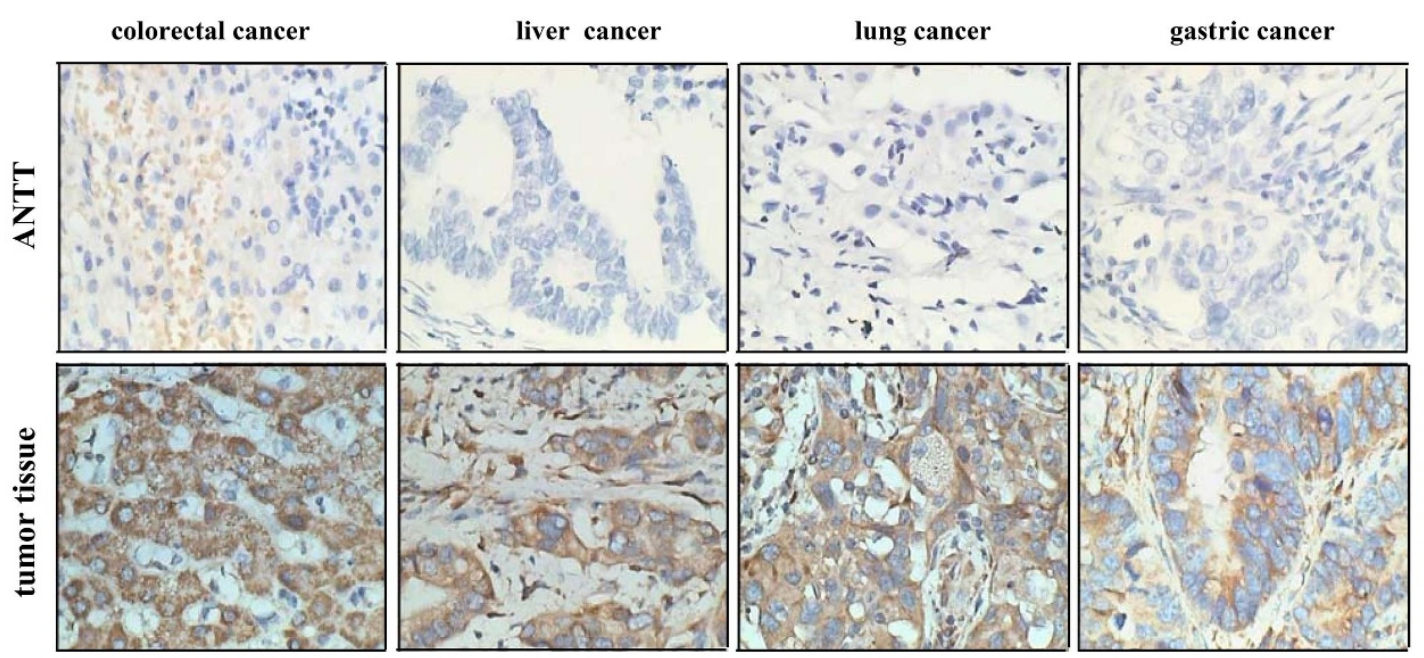

$\mathrm{D}$

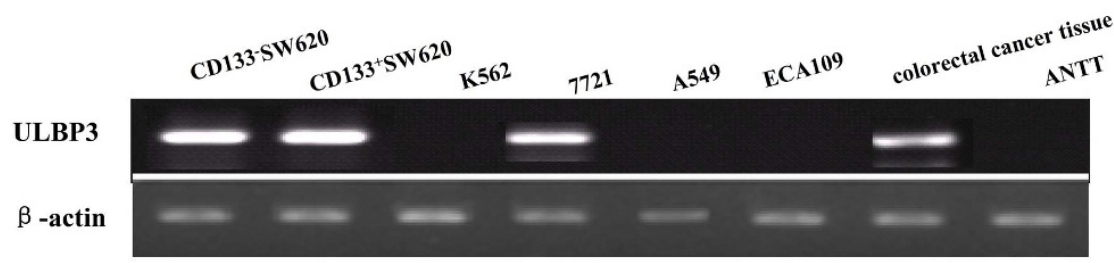

Figure 1 | ULBP3 expression in tumor cell lines and tumor tissues. (A) Surface expression of ULBP3 on CD133- ${ }^{-}$W620, CD133 ${ }^{+}$SW620, K562, 7721, A549, and ECA109 cell lines was measured by flow cytometry $(F C M)(n=3)$. ULBP3 protein was detected with the monoclonal antibody B2-F1-F1 $(5 \mu \mathrm{g} / \mathrm{ml})$ and a FITC-conjugated goat anti-mouse antibody. On each histogram, the red regions represent the isotype controls, and the black lines represent the marker of interest. (B) ULBP3 expression in single-cell suspensions of fresh tumor tissues from colorectal cancer ( $\mathrm{n}=3$ ), liver cancer $(\mathrm{n}=3)$, lung cancer $(\mathrm{n}=3)$, and gastric cancer $(\mathrm{n}=3)$ and paired adjacent non-tumor tissues (ANTT) was detected by FCM. Representative dot graphs are shown. The results are expressed as the mean \pm SEM. (C) Representative results from immunohistochemical staining for ULBP3 in colon, liver, lung, and stomach tumor tissues and ANTT are shown. Brown regions represent positive expression of ULBP3. Control indicates ANTT. The slides were analyzed under a $10 \times 40$ microscope equipped with a camera. (D) Total mRNA was extracted from tumor cell lines, colorectal tumor tissues and ANTT using TRIzol reagent. The mRNA expression of ULBP3 and $\beta$-actin was evaluated using RT-PCR. PCR products were visualized by gel electrophoresis in the presence of ethidium bromide. The data are representative of results obtained from at least 3 independent experiments.

and the percentage of NK cells in tumor tissues from 10 cancer patients ( 4 colorectal cancer, 3 lung cancer, and 3 gastric cancer). The percentage of infiltrating NK cells negatively correlated with ULBP3 expression ( $\mathrm{R}=-0.759, P=0.011)$. Representative data from 4 patients are shown in Figure 6A, and combined data are shown in Figure 6B. We then compared NK cytotoxicity in healthy individuals and in cancer patients with low $(<15 \mathrm{ng} / \mathrm{ml})$ or high $(>15 \mathrm{ng} / \mathrm{ml})$ levels of sULBP3. Freshly isolated NK cells from the peripheral blood mononuclear cells (PBMCs) of healthy individuals $(n=3)$ and pre-therapy cancer patients (3 colorectal cancer, 2 lung cancer, and 3 gastric cancer) were used as effecter cells. K562 cells served as target cells. The cytotoxicity of NK cells from cancer 

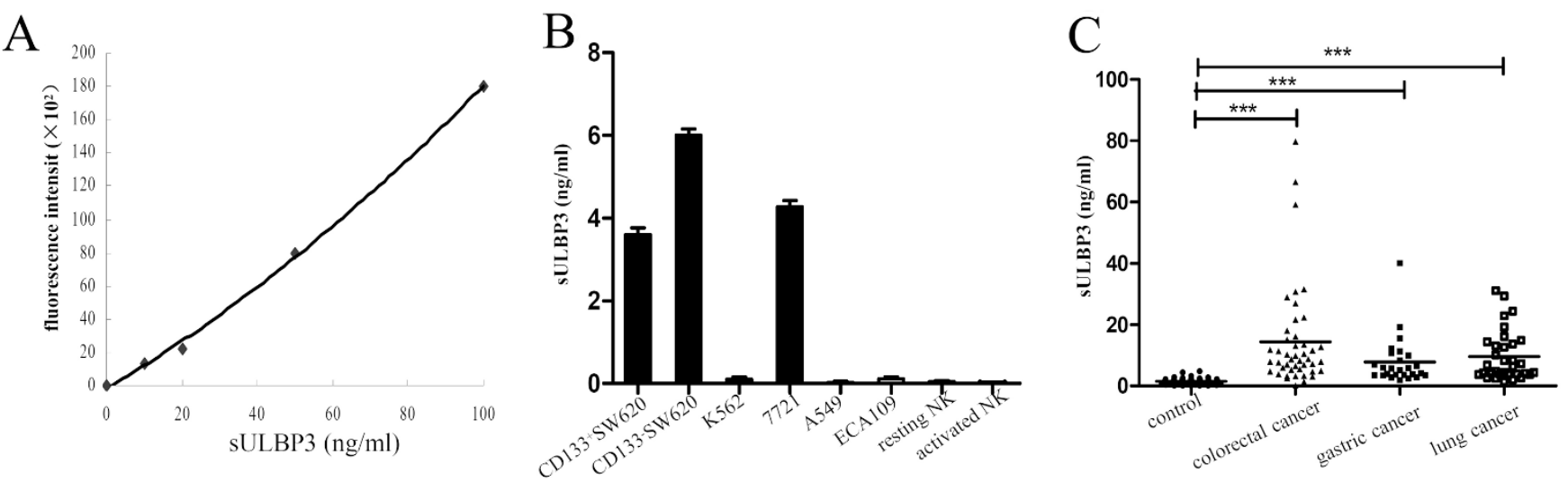

Figure 2 Detection of soluble ULBP3 (sULBP3) from tumor cells and in the serum of cancer patients. sULBP3 in serum samples from cancer patients or in the culture supernatant of tumor cell lines was measured by time-resolved fluoroimmunoassay (TRFIA), as described in the Materials and Methods section. (A) Standard curve for the detection of the sULBP3. (B) sULBP3 levels in the culture supernatant of cancer cell lines. (C) sULBP3 levels in the serum of healthy volunteers $(n=48)$ and patients with colorectal caner $(n=45)$, gastric cancer $(n=38)$, or lung cancer $(n=33)$. The results are expressed as the mean \pm SEM. ${ }^{* * *} P<0.001$.

patients with a low level of sULBP3 were decreased $(\mathrm{n}=5$, lysis $\%=$ $12.3 \pm 3.6 \%, P<0.05)$, compared with healthy individuals (lysis $\%=$ $27.9 \pm 2.4 \%)$. However, the cytotoxicity of NK cells from cancer patients with a high level of sULBP3 were normal $(\mathrm{n}=3$, lysis $=$ $25.4 \pm 3.7 \%)$.

Given that tumor cells generally express surface ULBP3 and sULBP3, we investigated whether an anti-ULBP3 antibody altered the cytotoxic activity of NK cells against ULBP3-expressing tumor cells. The lysis efficiency of the tumor cell lines CD133- SW620 (Figure $6 \mathrm{~B}$ ) and 7721 (Figure $6 \mathrm{C}$ ) at an $\mathrm{E}: \mathrm{T}$ ratio of $10: 1$ was enhanced in the presence of anti-ULBP3 monoclonal antibody (B2-F1-F1). Furthermore, when an antibody against CD16 receptor was added, the effect was blocked, suggesting that the elevated activity of NK cells resulted from ADCC. Consistently, the downregulation of NK cytotoxicity by sULBP3 in K562 (Figure 6D) or A549 (Figure 6E) cells lacking ULBP3 was also reversed by B2-F1-F1. Together, these results suggested that B2-F1-F1 abrogated the sULBP3-induced inhibition of NK cell cytotoxicity and induced surface ULBP3-mediated ADCC to enhance the cytotoxicity of NK cells.

\section{Discussion}

By regulating the innate and adaptive immune response through ligation of NKG2D, NKG2DLs are thought to be important for tumor initiation and development. For example, NKG2DL expression has been associated with prognosis, immunoediting, and the control of an immunological checkpoint that relies on NKG2Dmediated immune responses during the epithelial-to-mesenchymal transition $^{29,30}$. NKG2DL expression is of particular interest because previous experiments demonstrated that the NKG2D/NKG2DL system stimulates the immune surveillance of tumors ${ }^{12,31}$. Thus, NKG2DLs are potential targets for the development of novel immunotherapeutic anticancer regimens ${ }^{11}$. Although NKG2DLs such as MICA/B are widely expressed in many epithelial tumors and are associated with the regulation of NK and $\gamma \delta$ T cells, which play a key role in the elimination of tumor cells ${ }^{32}$, other NKG2DLs, such as ULBP1, ULBP2, and ULBP3, are not well characterized. In the

Table 1 | sULBP3 distribution in serum of cancer patients

\begin{tabular}{lccc} 
& Number & $<15 \mathrm{ng} / \mathrm{ml}(\%)$ & $\geq 15 \mathrm{ng} / \mathrm{ml}(\%)$ \\
\hline Colorectal cancer & 45 & $33(73.3)$ & $12(26.7)$ \\
Gastric cancer & 38 & $31(83.3)$ & $7(16.7)$ \\
Lung cancer & 33 & $28(82.3)$ & $5(17.7)$ \\
\hline
\end{tabular}

present study, we demonstrated that ULBP3, like other NKG2DLs, was widely expressed on primary tumor cells and tumor cell lines. The NK cell cytotoxicity elicited by surface ULBP3-expressing tumor cells was stronger than that elicited by non-expressing tumor cells, suggesting that the expression of surface ULBP3 on tumor cells is directly related to the activity of NK cells against tumor cells.

Previous studies have demonstrated that the shedding of sULBPs can alter the density of surface-expressed NKG2DLs. The soluble molecules have systemic functions that affect NK cell reactivity against tumor cells ${ }^{33}$. Relative to their surface-expressed counterparts, sULBPs may mediate similar, partially different, or opposite effects. In the present study, we demonstrated that ULBP3 was spontaneously released in soluble form from ULBP3-expressing cells. In a high proportion of the serum samples obtained from cancer patients, sULBP3 levels were elevated, indicating that the release of sULBP3 was not an artifact of in vitro tumor cell culture but a feature of human tumors. However, our findings differ from those of previous reports describing the effect of soluble NKG2DL on NK cell activity. In our study, only a low dose of sULBP3 $(<15 \mathrm{ng} / \mathrm{ml})$ decreased NK cytotoxicity by downregulating NKG2D expression on NK cells; a high dose of sULBP3 did not inhibit NK cell activity. These findings that a low concentration of sULBP3 downregulated NK cell activity in vitro were consistent with the discovery that more than $70 \%$ of cancer patients had a low concentration $(<15 \mathrm{ng} / \mathrm{ml})$ of serum sULBP3. Furthermore, the percentage of infiltrating NK cells negatively correlated with ULBP3 expression. These results suggest that a low concentration of sULBP3 contributes to the hyporesponsiveness of the immune system in cancer patients by decreasing NK cell activity, and they explain a general aspect of immunological dysfunction in cancer patients.

Serum levels of soluble MICA/B are elevated in patients with various types of cancer. Soluble MICA/B may be a diagnostic marker in patients with malignancies ${ }^{27,28}$. Soluble ULBP2 has also been detected in the serum of some patients with malignancies ${ }^{34}$. We have developed a method for detecting serum ULBP3. Our data demonstrated that the concentration of sULBP3 was significantly higher in colorectal, lung, and gastric cancer patients than in healthy individuals, Furthermore, cancer patients released a significant amount of sULBP3 in vivo. In future studies, it will be important to determine whether the level of serum ULBP3 in cancer patients correlates with the progression and outcome of malignancies, in order to assess ULBP3 as a potential tumor marker.

When analyzing tumor tissue samples from cancer patients, we found a negative correlation between the percentage of infiltrating NK cells and the level of ULBP3 expression. Surface-expressed and 


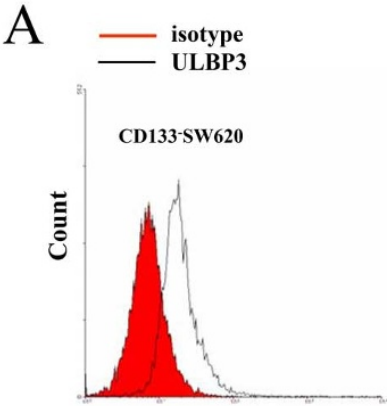

ULBP3

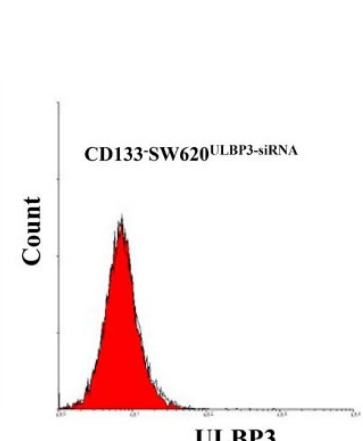

ULBP3
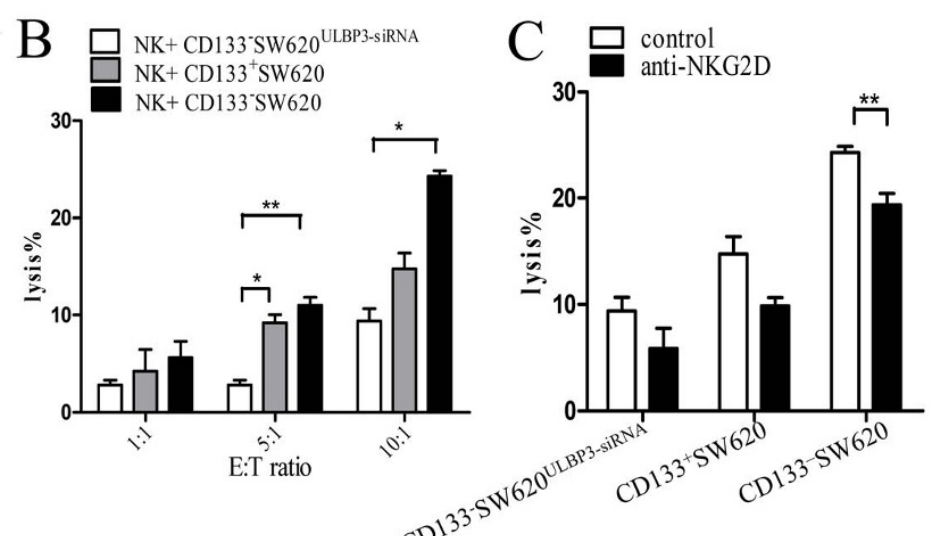

Figure 3 The effect of surface-expressed ULBP3 on NK cell activity. (A) The surface expression of ULBP3 on CD133-5W620 cells (left) or CD133-SW620 cells with ULBP3-siRNA transfection (right) was measured by FCM. (B-C) Effector NK cells were freshly isolated from healthy volunteers (normal NK cells), and 3 cell lines (ULBP3 siRNA-treated CD133-SW620, CD133+ SW620, and CD133- SW620) were used as target cells. The cytotoxicity of the NK cells was measured by LDH release after co-culture for $6 \mathrm{~h}$ at the indicated effector cell to target cell (E:T) ratios (B) and with or without the addition of anti-NKG2D $(5 \mu \mathrm{g} / \mathrm{ml})$ in co-cultures with an $\mathrm{E}:$ T ratio of $10: 1$ (C). The data are representative of results obtained from at least 3 independent experiments. The results are expressed as the mean \pm SEM. ${ }^{*} P<0.05,{ }^{*} P<0.01$.

soluble ULBP3 may have additive/synergistic effects and cooperate to reduce NK cell reactivity, thus enabling human tumor cells to evade NK-mediated surveillance. Given that tumor cells generally expressed surface ULBP3 and sULBP3, an anti-ULBP3 antibody could contribute to the activity of NK cells against ULBP3-expressing tumor cells. The neutralization of sULBP3 in patients might be a therapeutic strategy to enhance the immune response by promoting NK cell reactivity. Intriguingly, the addition of an anti-ULBP3 antibody prepared in our laboratory triggered ULBP3-mediated ADCC and increased NK cytotoxicity against ULBP3-expressing tumor cells, suggesting the antibody has immunotherapeutic value for cancer treatment.

\section{Methods}

Patient characteristics and clinical samples. The patients in this study were recruited from The Hospital Affiliated to Jiangsu University and were diagnosed through standard clinical and laboratory examinations. Healthy volunteers were selected based on normal physical and tumor marker examination. Peripheral blood
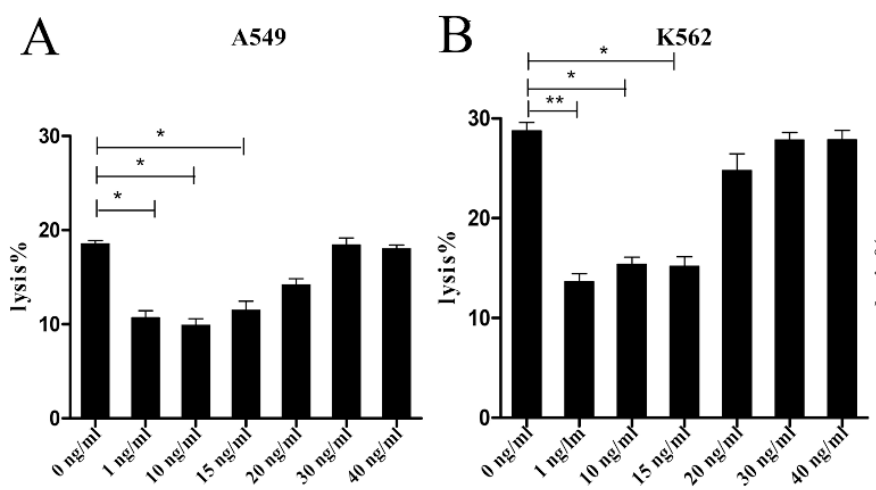

$\mathrm{C}$ 7721

$\mathrm{D}$ CD133-SW620
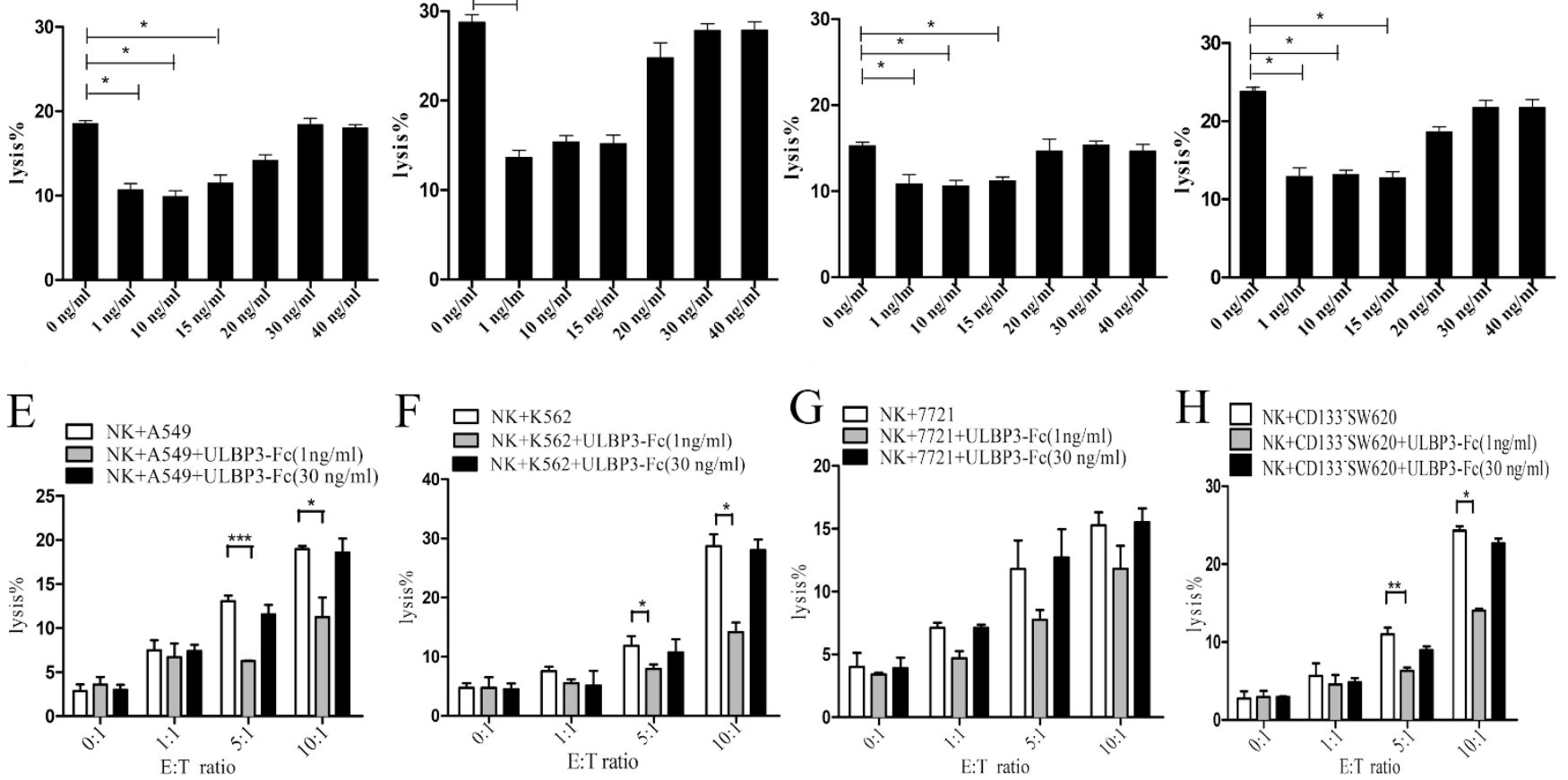

Figure 4 The effect of sULBP3 on NK cell activity. A549 (A), K562 (B), 7721 (C), and CD133-SW620 (D) cells were co-cultured with NK cells, with or without the indicated concentrations of ULBP3-Fc. NK cell cytotoxicity was measured by LDH release after co-culture for $6 \mathrm{~h}$ at an E: T ratio of 10:1. A549 (E), K562 (F), $7721(\mathrm{G})$, and CD133-SW620 (H) were co-cultured with NK cells respectively at the indicated E: T ratios, with or without adding high $(30 \mathrm{ng} / \mathrm{ml})$ or low $(1 \mathrm{ng} / \mathrm{ml})$ concentration of ULBP3-Fc. The NK cell cytotoxicity was measured by LDH release after co-culture for $6 \mathrm{~h}$. Data are representative of the results obtained from at least three independent experiments. Results are expressed as means \pm SEM. ${ }^{*} P<0.05, * * P<0.01$, $* * * P<0.001$ 

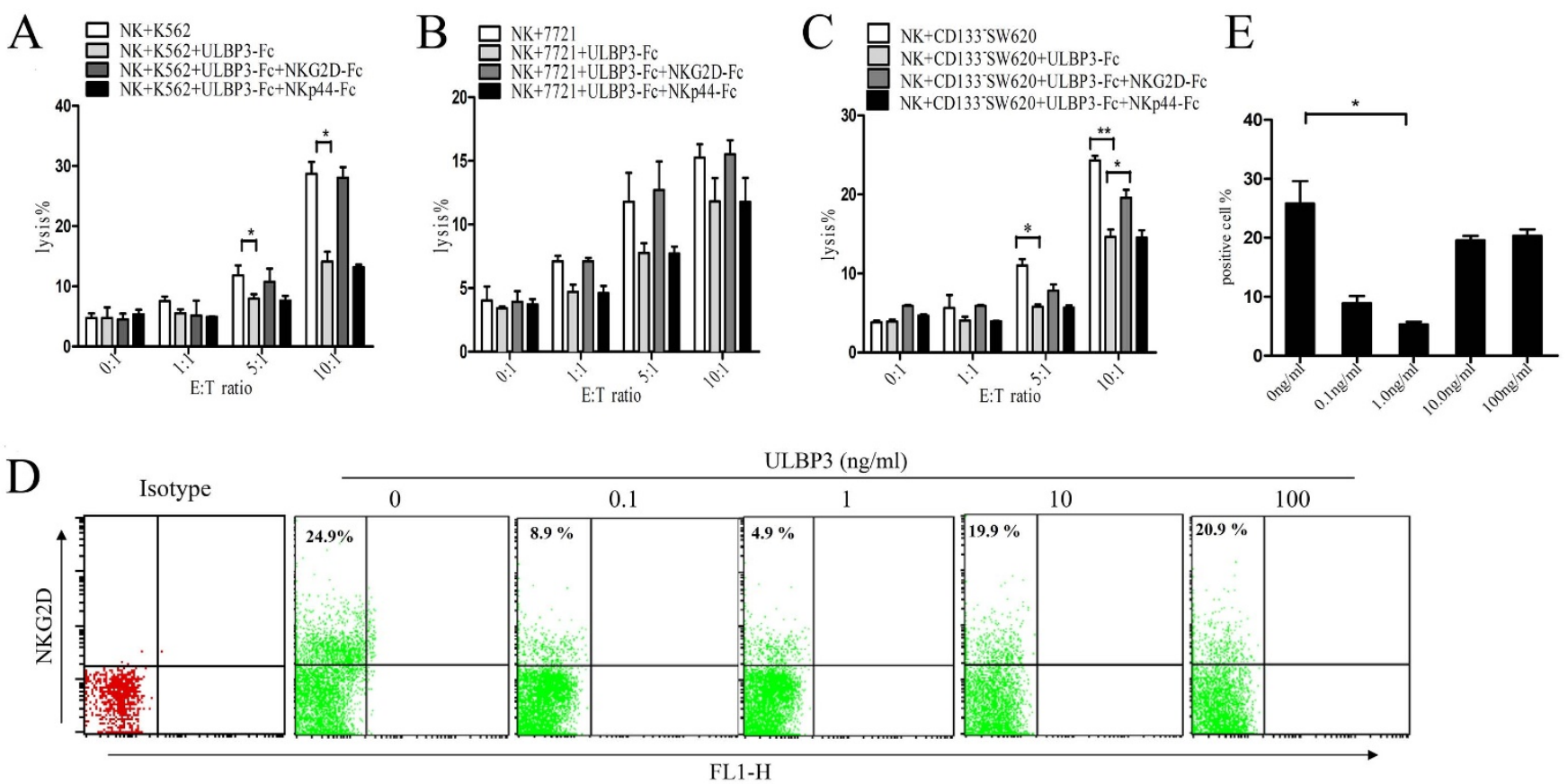

Figure $5 \mid$ NKG2D reverses the sULBP3-induced downregulation of NK cell cytotoxicity. K562 (A), 7721 (B), or CD133-5W620 (C) cells were cocultured with normal NK cells, with or without ULBP3-Fc (1 ng/ml), NKG2D-Fc (5 ng/ml), and NKp44-Fc (5 ng/ml). The cytotoxicity of NK cells was measured by LDH release after co-culture for $6 \mathrm{~h}$ at the indicated $\mathrm{E}$ : T ratios. (D) Normal NK cells were incubated with the indicated concentrations of ULBP3-Fc for 6 h, and NKG2D expression in NK cells was measured by FCM. A representative graph of NKG2D expression is shown. (E) Combined data of NKG2D expression are shown. The data are representative of results obtained from at least 3 independent experiments. The results are expressed as the mean \pm SEM. $* P<0.05, * * P<0.01$.

samples were obtained from patients before therapy. Serum was frozen at $-80^{\circ} \mathrm{C}$ for subsequent analysis. Fresh tumor tissue and ANTT were obtained during surgery from cancer patients before they received treatment. The tissue was cleaved and digested with collagenase and pancreatin. Single tumor cell suspensions were washed with PBS and resuspended. All patients and healthy volunteers provided written informed consent in accordance with the guidelines of the Ethics Committee of The Hospital Affiliated to Jiangsu University. This study has been approved by the Ethics
Committee of The Hospital Affiliated to Jiangsu University, and was performed accordance with the ethical standards laid down in the 1964 declaration of Helsinki and all subsequent revisions.

Cell culture and purification. The human tumor cell lines CD133+SW620, CD133-SW620 (colorectal cancer line), K562 (erythroleukemia cell line), 7721 (liver cancer cell line), A549 (lung cancer cell line), and ECA109 (esophageal carcinoma cell
A

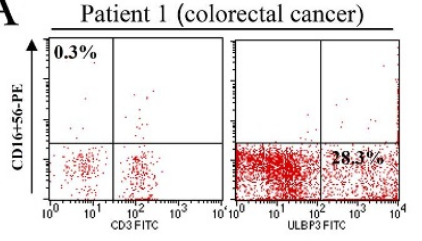

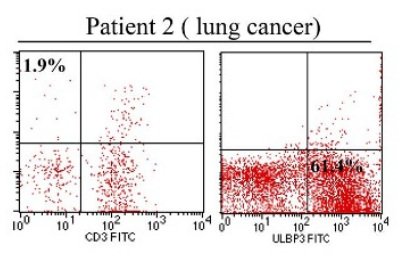

D

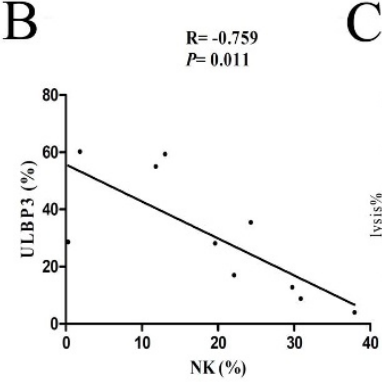

C

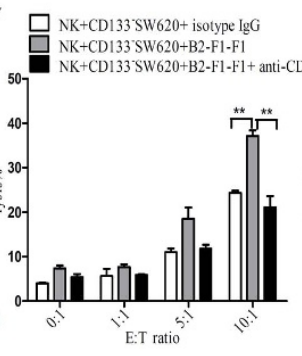

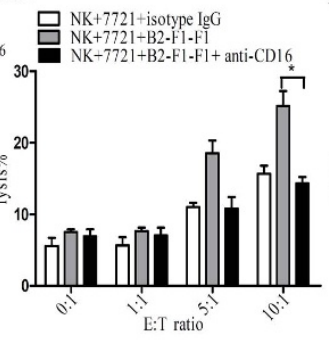

Patient 3 (gastric cancer)

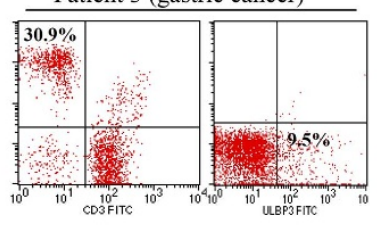

E

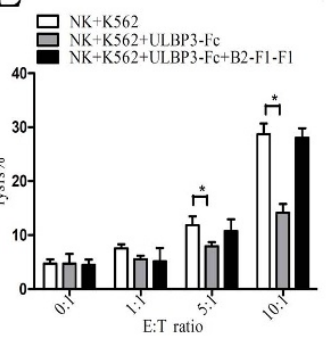

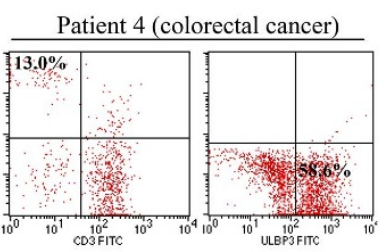

F

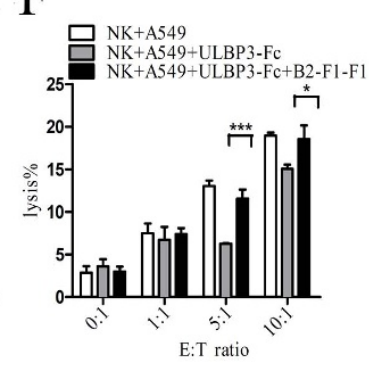

Figure 6 | Anti-ULBP3 monoclonal antibody enhances the activity of NK cells from cancer patients via ADCC. ULBP3 expression and the percentage of infiltrating NK cells in tumor tissues from 10 patients ( 4 colorectal cancer, 3 lung cancer, and 3 gastric cancer) were determined by FCM. A representative graph is shown in (A). The relationship between ULBP3 expression and infiltrating NK cells in tumor tissues is shown in (B). Normal NK cells were co-cultured with CD133-SW620 (C) or 7721 (D) cells at the indicated E:T ratios, and cytotoxicity was measured by LDH release after 6 h of co-culture, with or without the addition of isotype IgG, anti-ULBP3 (B2-F1-F1), and/or anti-CD16 (5 $\mu$ g/ml). K562 (E) or A549 (F) cells were co-cultured with normal NK cells, and cytotoxicity was measured by LDH release after $6 \mathrm{~h}$ of co-culture at the indicated $\mathrm{E}$ : T ratios, with or without the addition of ULBP3-Fc $(1 \mathrm{ng} / \mathrm{ml})$ and/or B2-F1-F1 $(5 \mu \mathrm{g} / \mathrm{ml})$. The data are representative of results obtained from at least 3 independent experiments. The results are expressed as the mean \pm SEM. $* P<0.05,{ }^{* *} P<0.01, * * * P<0.001$. 
line) were cultured in RPMI 1640 (Life Technologies, Gaithersburg, MD, USA) supplemented with $10 \%$ fetal bovine serum (FBS) at $37^{\circ} \mathrm{C}$ in an atmosphere containing $5 \% \mathrm{CO}_{2}$. NK cells were isolated from peripheral blood by negative selection using the NK Cell Isolation kit (Miltenyi Biotec, Bergisch Gladbach, Germany) according to the manufacturer's instructions. Experiments were performed when the purity of the NK cells was more than $90 \%$, as determined by FCM.

Reagents and antibodies. Human recombinant NKG2D-Fc (1299-NK), human recombinant NKp44-Fc, recombinant human ULBP3-Fc, and anti-CD16 (clone 245536) monoclonal antibody were purchased from R\&D Systems. FITC-mouse isotype IgG1, PE-mouse isotype IgG1, CD3-PE/CD (16 + 56)-FITC, and CD45-APC were purchased from Beckman. NKG2D-PE (clone 5C6) was purchased from eBioscience. The anti-NKG2D antibody (clone BAT221) was from Miltenyi Biotec. Anti-ULBP3 monoclonal antibodies (clones B2-F1-F1 and B4-C5-D11) were prepared in our laboratory ${ }^{35}$. CD56 microbeads were purchased from Miltenyi Biotech. Pancreatin and FITC-conjugated goat anti-mouse IgG $(\mathrm{H}+\mathrm{L})(\mathrm{A} 0568)$ were purchased from Beyotime, China. Collagenase (C-0130) was from SigmaAldrich.

Transfection with ULBP3-siRNA. ULBP3 expression was knocked down by transfecting cells with ULBP3 siRNA (sc-43006; Santa Cruz Biotech). CD133-SW620 cells $\left(5 \times 10^{5}\right)$ were plated in 6-well plates for $18 \mathrm{~h}$ and then transfected with $8 \mu \mathrm{l}$ of ULBP3 siRNA using Lipofector 2000 (Beyotime) in serum free medium for $5 \mathrm{~h}$. Thereafter, the medium was replaced with serum-supplemented medium for $24 \mathrm{~h}$. The cells were then used for functional assays.

FCM analysis. Cells $\left(1 \times 10^{5}\right)$ were suspended in PBS containing 2\% FBS for $10 \mathrm{~min}$ to block nonspecific binding sites. To determine the percentage of lymphocyte subsets, the cells were then incubated at $4{ }^{\circ} \mathrm{C}$ for $30 \mathrm{~min}$ with a combination of the following antibodies: NKG2D-PE, ULBP3-FITC, CD3-FITC/CD $(16+56)-P E$, and CD45-APC. For indirect staining, cells were washed twice with PBS and then incubated for $20 \mathrm{~min}$ at $4^{\circ} \mathrm{C}$ with FITC-conjugated goat anti-mouse IgG. Compensation was set with single-stained samples, low forward scatter elements (RBC and debris) were excluded from the analysis, and 10,000 events were collected and analyzed using a FACSAria cytometer (BD Biosciences).

Polymerase chain reaction (PCR). The following PCR primers were used: ULBP3, forward 5' - ATTCTTCCGTACCTGCTATT-3' and reverse 5' -GCTATCCTTCTCCCACTTCT- ${ }^{\prime} ; \beta$-actin, forward $5^{\prime}$-CACGAAACTACCTTCAACTAA- ${ }^{\prime}$ and reverse $5^{\prime}$-CATACTCCTGCTTGCTTGCTGATC-3'. ULBP3 gene expression was normalized to $\beta$-actin expression. Total RNA from colorectal tumor cell lines, tumor tissues, and ANTT was extracted using TRIzol after $48 \mathrm{~h}$ of cultivation. cDNA was obtained by reverse transcription of mRNA and used as the template for PCR. The PCR program consisted of $95^{\circ} \mathrm{C}$ for $4 \mathrm{~min}$, followed by 25 cycles of $94^{\circ} \mathrm{C}$ for $30 \mathrm{~s}$, $57^{\circ} \mathrm{C}$ for $30 \mathrm{~s}$, and $27^{\circ} \mathrm{C}$ for $40 \mathrm{~s}$, with a final extension at $72^{\circ} \mathrm{C}$ for $10 \mathrm{~min}$. All samples were run in triplicate.

Immunohistochemistry. Colorectal cancer tissues, liver cancer tissues, lung cancer tissues, and gastric cancer tissues were obtained from the department of pathology in The Hospital Affiliated to Jiangsu University. Tissue sections were treated with $0.3 \%$ hydrogen peroxidase for $5 \mathrm{~min}$ and blocked for $30 \mathrm{~min}$ with normal goat serum at room temperature. Anti-ULBP3 monoclonal antibody (B2-FI-FI) $(1: 200)$ was applied to the blocked sections and incubated overnight at $4^{\circ} \mathrm{C}$. The sections were incubated for $30 \mathrm{~min}$ at $37^{\circ} \mathrm{C}$ with HRP-labeled goat anti-mouse IgG antibody $(1: 2000)$, and the signals were developed with diaminobenzidine tetrahydrochloride solution. The sections were viewed with an Olympus Ax-70 DMC Ie CCD camera connected to a PC monitor.

Cytotoxicity assay. NK cell cytotoxic activity against the tumor cells was determined by measuring the amount of lactate dehydrogenase (LDH) released from the target cells. A commercial LDH cytotoxicity kit (Beyotime) was used according to the manufacturer's instructions. The maximum LDH release was determined by lysing target cells for $30 \mathrm{~min}$ using the lysis buffer provided with the assay and measuring $\mathrm{LDH}$ in the culture medium. Absorbance for the colorimetric reaction was measured at a wavelength of $490 \mathrm{~nm}$, with a reference wavelength of $655 \mathrm{~nm}$, using a Model 550 microplate reader (Bio-Rad, Hercules, CA, USA). The specific lysis for each effector to target cell $(\mathrm{E}: \mathrm{T})$ ratio was calculated with the following formula: $\%$ specific lysis = $[($ experimental release - spontaneous release)/(maximum release - spontaneous release) $] \times 100$.

Time-resolved fluoroimmunoassay for measuring sULBP3 levels in the serum. The wells of a 96-well plate were coated with the anti-ULBP3 monoclonal antibody B2-F1-F1 $(1 \mu \mathrm{g} /$ well $)$ in coating buffer overnight at $4{ }^{\circ} \mathrm{C}$. Human recombination ULBP3-Fc was diluted to different concentrations for use as a standard. Serum samples and tumor cell culture suspensions were added at $100 \mu \mathrm{l} /$ well and incubated at room temperature with shaking for $1 \mathrm{~h}$ and washed 6 times with buffer. Next, $\mathrm{Eu}^{3+}$ -labeled ULBP3 monoclonal antibody B4-C5-D11 (0.1 ng/well) was added, incubated at room temperature with shaking for $1 \mathrm{~h}$, and washed 6 times with buffer. Accentuation buffer was then added at $100 \mu \mathrm{l} /$ well, incubated with shaking for $0.5 \mathrm{~h}$, and washed 6 times. The $\mathrm{Eu}^{3+}$ fluorescence intensity was measured using an
AutoDELFIA 1235 immunoassay system (EG\&G Wallac), and data were analyzed using the MultiCalc software.

Statistics analysis. The results are expressed as the mean \pm SEM. Comparisons between 2 groups were performed with Student's $t$-test. Differences among groups were assessed using one-way analysis of variance followed by the Tukey post hoc multiple comparisons test. $P<0.05$ was considered statistically significant.

1. Topalian, S. L., Weiner, G. J. \& Pardoll, D. M. Cancer immunotherapy comes of age. J Clin Oncol 29, 4828-4836 (2011).

2. Movsas, B. et al. Randomized trial of amifostine in locally advanced non-small-cell lung cancer patients receiving chemotherapy and hyperfractionated radiation: radiation therapy oncology group trial 98-01. J Clin Oncol 23, 2145-2154 (2005).

3. Ljunggren, H. G. \& Malmberg, K. J. Prospects for the use of NK cells in immunotherapy of human cancer. Nat Rev Immunol 7, 329-339 (2007).

4. Moretta, L. \& Moretta, A. Unravelling natural killer cell function: triggering and inhibitory human NK receptors. EMBO J 23, 255-259 (2004).

5. Farag, S. S. \& Caligiuri, M. A. Human natural killer cell development and biology. Blood Rev 20, 123-137 (2006).

6. Jamieson, A. M. et al. The role of the NKG2D immunoreceptor in immune cell activation and natural killing. Immunity 17, 19-29 (2002).

7. Raulet, D. H. Roles of the NKG2D immunoreceptor and its ligands. Nat Rev Immunol 3, 781-790 (2003).

8. Groh, V. et al. Cell stress-regulated human major histocompatibility complex class I gene expressed in gastrointestinal epithelium. Proc Natl Acad Sci U S A 93, 12445-12450 (1996).

9. Pende, D. et al. Role of NKG2D in tumor cell lysis mediated by human NK cells: cooperation with natural cytotoxicity receptors and capability of recognizing tumors of nonepithelial origin. Eur J Immunol 31, 1076-1086 (2001).

10. Cosman, D. et al. ULBPs, novel MHC class I-related molecules, bind to CMV glycoprotein UL16 and stimulate NK cytotoxicity through the NKG2D receptor. Immunity 14, 123-133 (2001)

11. Ullrich, E., Koch, J., Cerwenka, A. \& Steinle, A. New prospects on the NKG2D/ NKG2DL system for oncology. Oncoimmunology 2, e26097 (2013)

12. Diefenbach, A., Jensen, E. R., Jamieson, A. M. \& Raulet, D. H. Rae1 and H60 ligands of the NKG2D receptor stimulate tumour immunity. Nature 413, 165-171 (2001).

13. Cerwenka, A., Baron, J. L. \& Lanier, L. L. Ectopic expression of retinoic acid early inducible-1 gene (RAE-1) permits natural killer cell-mediated rejection of a MHC class I-bearing tumor in vivo. Proc Natl Acad Sci U S A 98, 11521-11526 (2001).

14. Carayannopoulos, L. N. et al. Ligands for murine NKG2D display heterogeneous binding behavior. Eur J Immunol 32, 597-605 (2002).

15. O'Callaghan, C. A., Cerwenka, A., Willcox, B. E., Lanier, L. L. \& Bjorkman, P. J. Molecular competition for NKG2D: H60 and RAE1 compete unequally for NKG2D with dominance of H60. Immunity 15, 201-211 (2001).

16. Li, P. et al. Complex structure of the activating immunoreceptor NKG2D and its MHC class I-like ligand MICA. Nat Immunol 2, 443-451 (2001).

17. Bauer, S. et al. Activation of NK cells and T cells by NKG2D, a receptor for stressinducible MICA. Science 285, 727-729 (1999).

18. Champsaur, M. \& Lanier, L. L. Effect of NKG2D ligand expression on host immune responses. Immunol Rev 235, 267-285 (2010).

19. Rolle, A. et al. Effects of human cytomegalovirus infection on ligands for the activating NKG2D receptor of NK cells: up-regulation of UL16-binding protein (ULBP) 1 and ULBP2 is counteracted by the viral UL16 protein. J Immunol 171, 902-908 (2003).

20. Pende, D. et al. Major histocompatibility complex class I-related chain A and UL16-binding protein expression on tumor cell lines of different histotypes: analysis of tumor susceptibility to NKG2D-dependent natural killer cell cytotoxicity. Cancer Res 62, 6178-6186 (2002).

21. de Kruijf, E. M. et al. NKG2D ligand tumor expression and association with clinical outcome in early breast cancer patients: an observational study. BMC Cancer 12, 24 (2012).

22. Hilpert, J. et al. Comprehensive analysis of NKG2D ligand expression and release in leukemia: implications for NKG2D-mediated NK cell responses. J Immunol 189, 1360-1371 (2012).

23. Groh, V., Wu, J., Yee, C. \& Spies, T. Tumour-derived soluble MIC ligands impair expression of NKG2D and T-cell activation. Nature 419, 734-738 (2002).

24. Sconocchia, G. et al. The antileukemia effect of HLA-matched NK and NK-T cells in chronic myelogenous leukemia involves NKG2D-target-cell interactions. Blood 106, 3666-3672 (2005).

25. Doubrovina, E. S. et al. Evasion from NK cell immunity by MHC class I chainrelated molecules expressing colon adenocarcinoma. J Immunol 171, 6891-6899 (2003).

26. Jinushi, M. et al. Impairment of natural killer cell and dendritic cell functions by the soluble form of MHC class I-related chain A in advanced human hepatocellular carcinomas. J Hepatol 43, 1013-1020 (2005).

27. Holdenrieder, S. et al. Soluble MICA in malignant diseases. Int J Cancer 118, 684-687 (2006).

28. Wu, J. D. et al. Prevalent expression of the immunostimulatory MHC class I chain-related molecule is counteracted by shedding in prostate cancer. J Clin Invest 114, 560-568 (2004). 
29. McGilvray, R. W. et al. NKG2D ligand expression in human colorectal cancer reveals associations with prognosis and evidence for immunoediting. Clin Cancer Res 15, 6993-7002 (2009).

30. Lopez-Soto, A. et al. Regulation of NKG2D signaling during the epithelial-tomesenchymal transition. Oncoimmunology 2, e25820 (2013).

31. Lanier, L. L. A renaissance for the tumor immunosurveillance hypothesis. Nat Med 7, 1178-1180 (2001).

32. Groh, V. et al. Broad tumor-associated expression and recognition by tumorderived gamma delta T cells of MICA and MICB. Proc Natl Acad Sci U S A 96, 6879-6884 (1999).

33. Song, H., Kim, J., Cosman, D. \& Choi, I. Soluble ULBP suppresses natural killer cell activity via down-regulating NKG2D expression. Cell Immunol 239, 22-30 (2006).

34. Waldhauer, I. \& Steinle, A. Proteolytic release of soluble UL16-binding protein 2 from tumor cells. Cancer Res 66, 2520-2526 (2006).

35. Mao, C. et al. A monoclonal antibody against human UL16-binding protein 3. Hybridoma (Larchmt) 31, 203-208 (2012).

\section{Acknowledgments}

We thank Dr. Yichuan Xiao for critical reading. This work was supported by grants from the National Natural Science Foundation of China (81370889, 81270801 and 81370965), the
Natural Science Foundation of Jiangsu Province (BK20131248), and the Technology Commission of Zhenjiang Municipality (SH2012023, SH2013031).

\section{Author contributions}

X.M., Y.Z. and P.J. performed the experiments and analyzed the data. C.M. designed the project and wrote the manuscript. Y.Z. and T.Z. edited various parts of the manuscript. Q.J., X.C., H.L. and T.Z. helped with the experimental design. G.Y. and D.C. supervised the data analysis and edited the manuscript. All authors reviewed the manuscript.

\section{Additional information}

Competing financial interests: The authors declare no competing financial interests.

How to cite this article: Mou, X. et al. The Regulatory Effect of UL-16 Binding Protein-3 Expression on the Cytotoxicity of NK Cells in Cancer Patients. Sci. Rep. 4, 6138; DOI:10.1038/srep06138 (2014).

This work is licensed under a Creative Commons Attribution 4.0 International License. The images or other third party material in this article are included in the article's Creative Commons license, unless indicated otherwise in the credit line; if the material is not included under the Creative Commons license, users will need to obtain permission from the license holder in order to reproduce the material. To view a copy of this license, visit http://creativecommons.org/licenses/by/4.0/ 